\title{
Antihypertensive Drugs and Cancer: Simultaneously Development of Choroidal Melanoma and Colon Carcinoma after Administration with Valsartan/ Hydrochlorothiazide
}

\author{
Georgi Tchernev*, Gavrail Poterov \\ Onkoderma- Clinic for Dermatology, Venereology and Dermatologic Surgery, General Skobelev, Sofia, Bulgaria
}

\author{
Received: October 21, 2020; Accepted: October 24, 2020; Published: October 27, 2020 \\ *Corresponding author: Professor Georgi Tchernev, PhD, Onkoderma-Clinic for Dermatology, Venereology and Dermatologic Surgery, Sofia, \\ Bulgaria, Tel. No: 00359885588424; E-mail: georgi_tchernev@yahoo.de
}

\begin{abstract}
Recently published experimental data on the presence of different types of angiotensin receptors in melanoma tissue and in breast cancer, for example, are undoubtedly alarming and indicative because Sartan therapy (in certain groups of patients) may be a risk factor for the patients themselves. The reason for this is the ability of the specific group of drugs to promote carcinogenesis by blocking certain types of receptors, presented in certain types of tumors.

The available in vivo and in vitro data in the world literature on the influence of antihypertensive drugs (Sartans and Thiazide diuretics) on melanogenesis and development of other cancerous forms, although disputed, are numerous. Even more disturbing are the further data in the medical literature on the development of multiple tumors after administration of Sartans alone or in combination with hydrochlorothiazide. According to other literature data, even monotherapy with the already mentioned diuretic- hydrochlorothiazide, could be seen as a possible key or risk factor for the development of cutaneous melanoma. We present a clinical case of a 67-year-old patient with anamnestic data for systemic antihypertensive therapy with a combined preparation containing Valsartan / hydrochlorothiazide $160 \mathrm{mg} / 12.5 \mathrm{mg}$ for the period from the end of 2011 to March, 2020, came on occasion of a prophylactic dermatological examination. The examination found a pigmented lesion in the right torso, clinically and dermatoscopically suspected of thin cutaneous melanoma. According to the anamnesis and available documentation, it is clear that within this combined antihypertensive medication, the patient has developed two additional neoplasms in the recent past: choroidal melanoma and colorectal carcinoma successfully treated surgically.

To what extent, the shared data (on the occurrence of multiple tumors after combination treatment with antihypertensives such as sartans and thiazide diuretics) would lead to the necessary and desirable rethinking of therapeutic strategies for the treatment of hypertension and clarification of the mechanisms at molecular level, at this moment - remains unclear. But in practice it should be highly recommended.
\end{abstract}

Keywords: Melanoma; Melanogenesis; Antihypertensive Therapy; Valsartan; Sartans; Surgery; Hydrochlorothiazide

\section{Introduction}

A number of experimental studies in the world literature show that Sartans, depending on the type and location of angiotensin receptors in a particular tumor tissue, are able not only to block but also to potentiate carcinogenesis $[1,2]$. In particular with regard to melanoma, this could probably have fatal consequences due to the triggering of metastasis, for example $[1,2]$. The pathogenesis of cutaneous melanoma is still considered incompletely understood. Clinicians are confronting daily with new and new pathogenetic dilemmas such as druginduced / mediated carcinogenesis or melanogenesis, and in particular the role of the renin-angiotensin system in melanoma tissue [2].

\section{Clinical Case}

The anamnesis is taken according to the patient's data and the accompanying documentation. We present a clinical case of a 67-year-old patient who came for a prophylactic dermatological examination at the clinic to clarify the diagnosis of a lesion suspected clinically and dermatoscopically of a thin melanoma located in the torso on the right (Fig. 1a-1d). Arterial hypertension and hyperuricemia are the known comorbidities. A Systemic therapy is taken: Valsartan / Hydrochlorothiazide 160 / $12.5 \mathrm{mg}$ (with two different commercial preparations) according to a scheme (1-0-0)) from 2011 to 03.2020. Febuxostat $60 \mathrm{mg}$, once daily and Amlodipine $10 \mathrm{mg}$, once daily (up to 3 weeks ago). In November 2018, he conducted a consultative examination with an ophthalmologist on the occasion of deterioration of vision, and he was diagnosed with hyperopia, astigmatism and presbyopia, and the symptoms are about a year old. Four months later, in March 2019, a second consultative examination revealed severe melanosis of the conjunctiva of the right eye and macular degeneration. On this occasion, in April 2019, the patient conducted more in-depth ophthalmological examinations - orbital ultrasound and retinal fluorescein angiography. Ultrasound showed a solid lesion measuring 2.9 

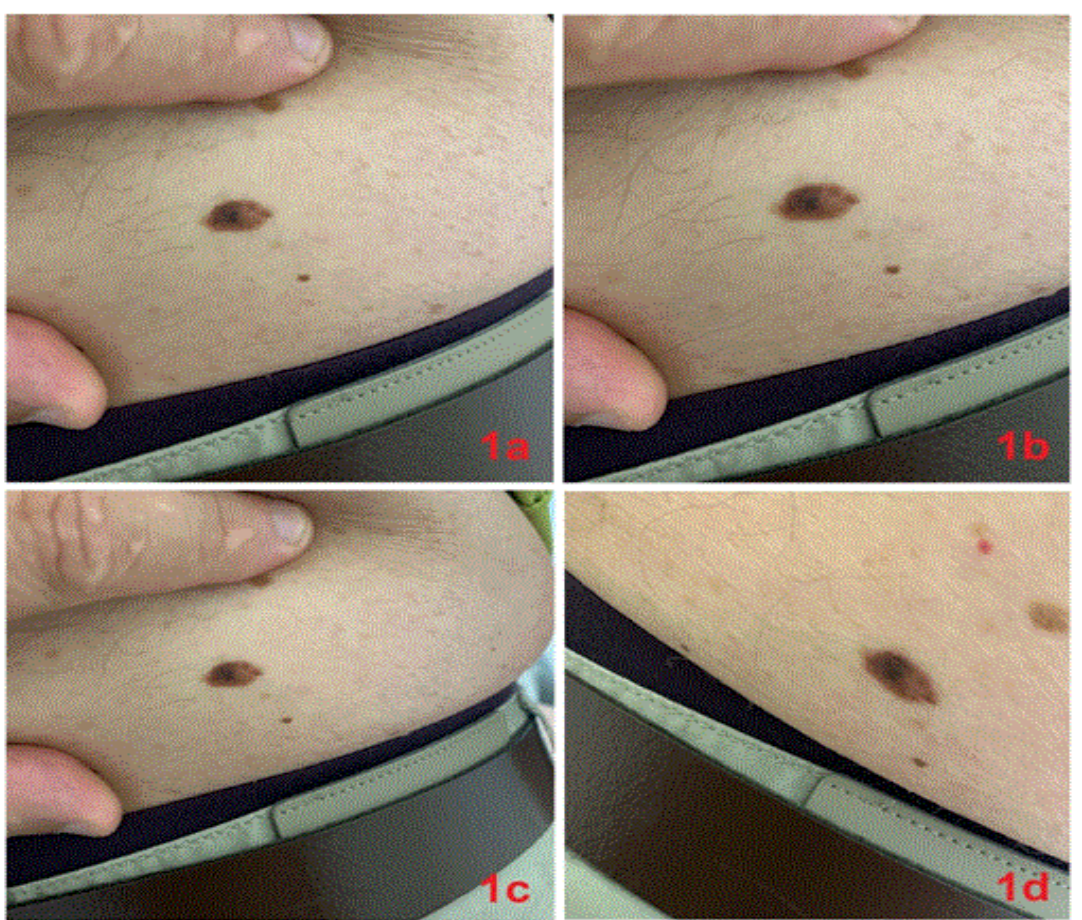

Figure 1a-1d: Pigmented lesion located on the right side of the torso, with clinical and dermatoscopic evidence of thin melanoma or melanoma in situ.

/ $5.9 \mathrm{~mm}$, apparent excavation, inhomogeneous structure, diagnosed with intraorbital tumor and suspected metastasis or hemangioma. Retinal angiography showed the presence of a rounded tumoriform lesion localized in the papillary area, which emerged as hyperfluorescent (Fig.2a-2d). The lesion is monitored periodically every 3 months and for 8 months almost doubles in size - from 2.9 / $5.9 \mathrm{~mm}$ to 6.11 / $9.60 \mathrm{~mm}$ in December 2019, when control studies clearly point in favor of malignant melanoma of the choroid (Fig. .3b-3f). Surgical treatment was also performed on February 25, 2020, with enucleation of the right bulb, followed by brachytherapy and placement of an eye prosthesis. The histological examination showed evidence of choroidal melanoma with a tumor thickness of $4 \mathrm{~mm}$, no infiltration of the optic nerve, with ocular melanocytosis. Regarding the underlying disease, the patient underwent screening ultrasound examinations of the liver and abdomen at 3 months and in June 2020 was diagnosed clinically and subsequently verified histologically tubulo-villous adenoma with severe dysplasia in the sigmoid colon. On June 17, 2020, a sigmoidectomy was performed with the dissection of the loco-regional lymph nodes without evidence of metastases in them. Highly differentiated adenocarcinoma of the colon, infiltrating and the serosa, 21 lymph nodes without metastases was diagnosed. Stage G1pT4aN0Mx. The patient was referred to the regional oncology dispensary for follow-up every 3 months for the risk of developing metastases from uveal melanoma or colorectal adenocarcinoma.
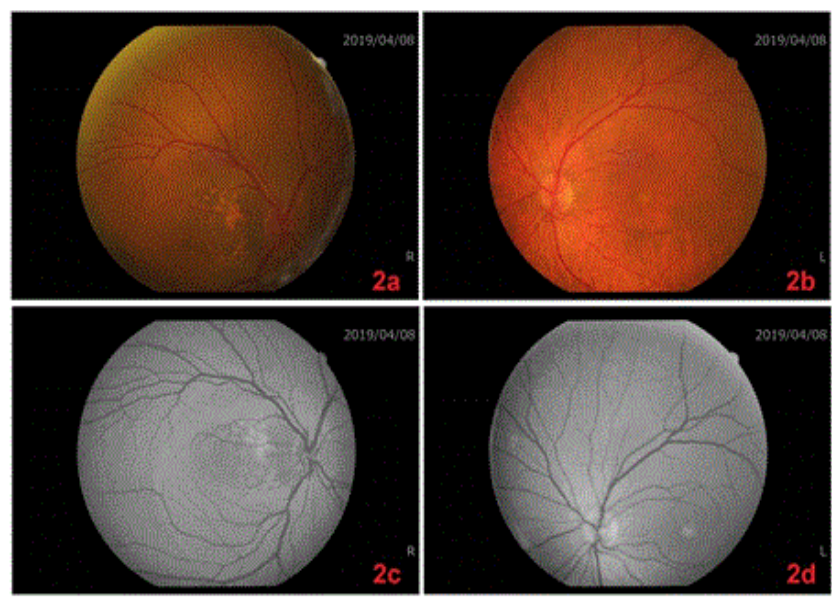

Figure 2: Retinal angiography, April 2019 - a / b - colored, c / d - fluorescent - in the papillary area there are data for a rounded lesion with an area of $2 \frac{1}{2} \mathrm{PD}$, which appears hyperfluorescent in the initial phases of X-ray diffraction with hyperfluorescent zones in the initial halves of the lesion, hyperfluorescence persists in the final phases and corresponds to the ophthalmoscopically observed atrophy of the pigment epithelium. Solid lesion with size 2.9 / $5.9 \mathrm{~mm}$, apparent excavation, inhomogeneous structure. The diagnosis is intraorbital tumor - metastasis or hemangioma. 


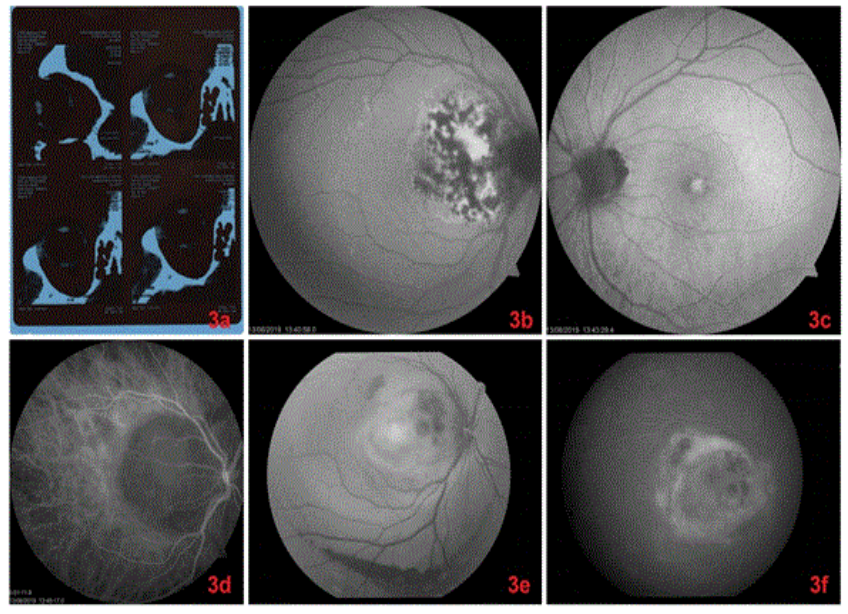

Figure 3a: CT of the orbits, hyperdense formation with dimensions $3 \mathrm{x}$ $5 \times 7 \mathrm{~mm}$. After intravenous contrast, a moderate and brief increase in the density of the lesion was observed. No evidence of exteriorizationretrobulbarly.

Figure 3b-f: Retinal angiography, December 2019 - the tumor has increased in size - 6.11 / $9.60 \mathrm{~mm}$, marked loboid shape, visible excavation, homogeneous structure, flat satellite retinal elevation. Despite the atypical localization, the data shows that probably is malignant melanoma of the choroid of the right bulb. A brachytherapy is recommended as an organ-preserving intervention.

\section{Discussion}

Numerous experimental (in vitro) data are available in the world literature for 1) on the one hand for the direct influence of Sartans on melanogenesis [1,2], which are supported by a serious dose of clinical (in vivo) data on the occurrence of melanoma after initiating treatment with the mentioned drugs [3-7]. The therapy with Sartans and Hydrochlorothiazide in combination could probably be seen to a certain point as problematic regarding the concomitant development of both melanocytic and non-melanocytic tumors or other solid tumors in combination with melanocytic one $[8,9]$. Hydrochlorothiazide monotherapy, even without a combination with Sartan, according to a number of authors, could definitely be somewhat risky in terms of the development of various forms of cutaneous melanoma $[10,11]$.

The combination of Hydrochlorothiazide with Sartan is likely to have an additional co-stimulating effect not only in terms of optimal treatment of hypertension, but also in terms of the formation or stimulation of carcinogenesis in the direction of melanoma or melanoma, but in combination with another type of a solid tumor for example [9].

It is for this reason that there is no doubt that there is a growing need for specific highly selective screening programs to assess the individual risk of developing melanoma, but also breast cancer and other tumors. These screening programs should be initiated prior to initiating therapy with Sartans / with or without combination with thiazide diuretics. Regardless of the assessment of individual risk, patients should undergo a serious clinical and paraclinical and hardware monitoring, with a view to early detection and treatment of possible tumor formations. An additional concern is that the Nitrosamines contained in Sartans as contaminants in the production process tend to induce up to 11 forms of cancer according to the FDA / EMA, including the colorectal adenocarcinoma described in our patient [4]. The presented case is interesting in terms of the possible development of two independent neoplasms for which Valsartan, taken as systemic therapy for about 9 years in combination with Hydrochlorothiazide, could have a possible key or mutually potentiating, triggering effect, co-stimulating role of the active ingredient in relation to the uveal and possible hypothetical second cutaneous melanoma (not operated so far, presented in figure (1a / d)) and possibly additional nitrosamines - probable in relation to colorectal adenocarcinoma ( a check for the presence of or lack of nitrosamines on the Bulgarian drug market turns out to be a serious dilemma or impossible at the time of our inquiry). From the accumulated scientific data in the world literature on the side effects of systemic therapy with Sartans, but also with Hydrochlorothiazide, their role in the treatment of cardiac diseases should be seriously reconsidered. The multiple development of tumors of different genesis should be seen as an alarming signal regarding the future clarification of this seemingly neglected or "deliberately overslept" by health systems and control authorities dilemma or issue.

\section{Conclusion}

In order to clarify as quickly as possible the potential carcinogenic effects of the active ingredients and / or cocarcinogenic impurities below in the form of nitrosamines, the following would be of paramount importance:

1) Testing of all sartans on the market for the presence of nitrosamines, which are currently described as possible triggers for colon cancer.

2) Experimental testing not only of Losartan, but also of other drugs from the group of Sartans, for which a similar or analogous carcinogenesis-stimulating effect is assumed: Irbesartan, Olmesartan, Telmisartan and Valsartan. These are drugs in the course of which cutaneous melanoma and other tumors have been detected in parallel or simultaneously.

3) Clarification under experimental conditions of the direct or indirect procarcinogenic effect of thiazide diuretics.

4) Testing various other tumors such as prostate cancer and adenocarcinoma of the colon for the presence or absence of angiotensin receptors in tumor tissue.

\section{References}

1. Olschewski DN, Hofschröer V, Nielsen N, Seidler DG, Schwab A, Stock C. The Angiotensin II Type 1 Receptor Antagonist Losartan Affects NHE1-Dependent Melanoma Cell Behavior. Cell PhysiolBiochem. 2018;45(6):2560-2576. doi:10.1159/000488274

2. Renziehausen A, Wang H, Rao B, Weir L, Nigro C, Lattanzio L, et al. The renin angiotensin system (RAS) mediates bifunctional growth regulation in melanoma and is a novel target for therapeutic intervention. Oncogene. 2019;38(13):2320-2336. doi:10.1038/ 
s41388-018-0563-y

3. Tchernev G, Temelkova I. Additional 4 cases of valsartan/irbesartaninduced melanomas? J BiolRegulHomeost Agents. 2019;33(3):911912.

4. Tchernev G, Temelkova I. Valsartan Induced Melanoma?! First Description in Medical Literature! Open Access Maced J Med Sci. 2018;6(12):2378-2380. doi:10.3889/oamjms.2018.517

5. Tchernev G, Temelkova I. Drug-Induced Melanoma: Irbesartan Induced Cutaneous Melanoma! First Description in the World Literature!. Open Access Maced J Med Sci. 2019;7(1):114-116. doi:10.3889/ oamjms.2019.042

6. Tchernev G, Temelkova I. Irbesartan Induced Cutaneous Melanoma! Second Case in the Medical Literature! Open Access Maced J Med Sci. 2019;7(1):121-123. doi:10.3889/oamjms.2019.043

7. Tchernev G, Temelkova I. Olmesartan/valsartan induced giant achromatic cutaneous melanoma: "modified" one-step surgical approach with favourable outcome. J BiolRegulHomeost Agents. 2019;33(6):1775-1777.
8. Malev V, Tchernev G. Dysplastic nevus and BCC development after antihypertensive therapy with Valsartan and Hydrochlorothiazide!? Clin Res Dermatol Open Access. 2019;6(5):1-2.

9. Tchernev G, Temelkova I. Valsartan/hydrochlorothiazide induced prostate carcinoma in a patient who subsequently developed melanoma. J Biol Regul Homeost Agents. 2019;33(4):1125-1127.

10.Schmutz JL. L'hydrochlorothiazide augmenterait le risque de mélanome [Hydrochlorothiazide appears to increase risk of melanoma]. Ann Dermatol Venereol. 2018;145(10):643-644. doi:10.1016/j.annder.2018.07.007

11. Pottegård A, Pedersen SA, Schmidt SAJ, Hölmich LR, Friis S, Gaist D. Association of Hydrochlorothiazide Use and Risk of Malignant Melanoma. JAMA Intern Med. 2018;178(8):1120-1122. doi:10.1001/ jamainternmed.2018.1652 